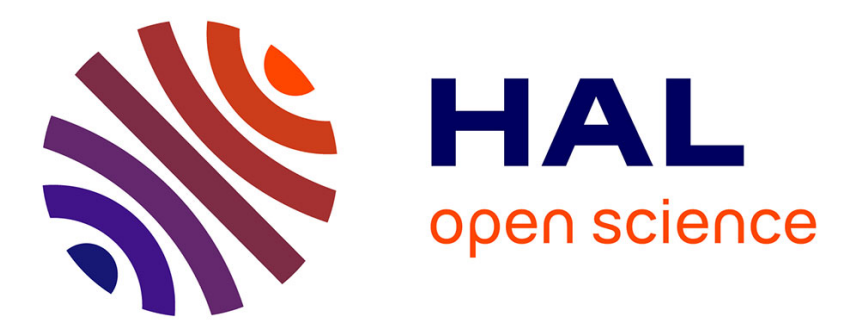

\title{
Degeneration to infinity may provide information about kinematics
}

\author{
Michel Coste, Nestor Djintelbe
}

\section{To cite this version:}

Michel Coste, Nestor Djintelbe. Degeneration to infinity may provide information about kinematics. Lenarčič; Jadran and Siciliano; Bruno. Advances in Robot Kinematics 2020, Springer International Publishing, pp.234-241, 2022, 978-3-030-50975-0. hal-02443359

\section{HAL Id: hal-02443359 https://hal.science/hal-02443359}

Submitted on 17 Jan 2020

HAL is a multi-disciplinary open access archive for the deposit and dissemination of scientific research documents, whether they are published or not. The documents may come from teaching and research institutions in France or abroad, or from public or private research centers.
L'archive ouverte pluridisciplinaire HAL, est destinée au dépôt et à la diffusion de documents scientifiques de niveau recherche, publiés ou non, émanant des établissements d'enseignement et de recherche français ou étrangers, des laboratoires publics ou privés. 


\title{
Degeneration to infinity may provide information about kinematics
}

\author{
Michel Coste and Nestor Djintelbe
}

\begin{abstract}
We propose to degenerate the kinematics of a mobile platform by letting the lengths of its legs tend to infinity. The rationale for this approach is that the degenerate situation is simpler to analyze and may provide relevant information for the kinematic analysis of the platform. We implement this approach in the framework of the Study model. We show its usefulness on the example of the general operation mode of the Tsai 3-UPU platform, whose kinematic analysis is rather difficult.
\end{abstract}

Key words: Study quadric, degeneration, 3-UPU platform.

\section{Introduction}

The Study model has proved very useful for the algebraic modelization of problems in robotics and their resolution in computer algebra systems. It realizes the group of rigid motions as a subset of a 6-dimensional quadric (the Study quadric $S$ ) in the 7dimensional real projective space. Precisely, the group of rigid motions is identified with the complement in $S$ of a 3-plane $E$ contained in $S$. For the Study model and its use in kinematics, one can see [3-5].

The exceptional plane $E$ can be seen as the boundary of the group of rigid motions in $S$, but there is no useful interpretation of its points as degenerate rigid motions. So we modify the Study model in order to have a boundary of dimension 5 whose points can be seen as degenerate rigid motions with infinite translation part. This modification is described in Section 2. An alternative modification is described in [2], using the notion of blowing-up.

Michel Coste

IRMAR, Université Rennes 1, France, e-mail: michel.coste@univ-rennes1.fr Nestor Djintelbe

Université Assane Seck, Ziguinchor, Sénégal, e-mail: djin_nestor@yahoo.fr 
The purpose of the introduction of a meaningful boundary for the group of rigid motions is to obtain information on the kinematics of the robots as the lengths of their legs are large enough. We have chosen to illustrate this approach with the example of the Tsai 3-UPU platform; a thorough kinematic analysis of this parallel manipulator has been done in [6]. We present a short survey in Section 3, insisting of the so-called general operation mode which is rather mysterious. In Section 4, we show that the degeneration of this general mode produces new relevant informations on its kinematic behaviour.

\section{The Study model and its modification}

We recall the Study model for the group of rigid motions and then define a modification of this model. This modification aims at obtaining a boundary which allows to degenerate rigid motions as the length of the translation vector tends to infinity.

\subsection{The Study model}

The Study model of the group of rigid motions is the quadric $S$ with equation

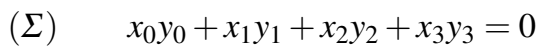

where $\left[x_{0}: \ldots: x_{3}: y_{0}: \ldots: y_{3}\right]$ are homogeneous coordinates in the real projective 7-space. We denote $\mathbf{x}=\left[x_{0}, x_{1}, x_{2}, x_{3}\right]^{\top}$ and $\mathbf{y}=\left[y_{0}, y_{1}, y_{2}, y_{3}\right]^{\top}$. We recall that to each point of this quadric outside of the exceptional 3-plane $E$ with equation $\mathbf{x}=\mathbf{0}$, we associate the rigid motion with rotation matrix

$$
\mathbf{R}(\mathbf{x})=\frac{1}{\|\mathbf{x}\|^{2}}\left[\begin{array}{ccc}
x_{0}^{2}+x_{1}^{2}-x_{2}^{2}-x_{3}^{2} & 2\left(x_{1} x_{2}-x_{0} x_{3}\right) & 2\left(x_{1} x_{3}+x_{0} x_{2}\right) \\
2\left(x_{1} x_{2}+x_{0} x_{3}\right) & x_{0}^{2}-x_{1}^{2}+x_{2}^{2}-x_{3}^{2} & 2\left(x_{2} x_{3}-x_{0} x_{1}\right) \\
2\left(x_{1} x_{3}-x_{0} x_{2}\right) & 2\left(x_{2} x_{3}+x_{0} x_{1}\right) & x_{0}^{2}-x_{1}^{2}-x_{2}^{2}+x_{3}^{2}
\end{array}\right]
$$

and translation vector

$$
\mathbf{t}(\mathbf{x}, \mathbf{y})=\frac{2}{\|\mathbf{x}\|^{2}}\left[\begin{array}{l}
x_{0} y_{1}-x_{1} y_{0}+x_{2} y_{3}-x_{3} y_{2} \\
x_{0} y_{2}-x_{1} y_{3}-x_{2} y_{0}+x_{3} y_{1} \\
x_{0} y_{3}+x_{1} y_{2}-x_{2} y_{1}-x_{3} y_{0}
\end{array}\right] \text { where }\|\mathbf{x}\|^{2}=x_{0}^{2}+x_{1}^{2}+x_{2}^{2}+x_{3}^{2}
$$

\subsection{Modification of the Study model}

The modification of $S$ with which we shall work is a subset $\widetilde{S}$ of the real 9dimensional affine space with coordinates $w_{0}, w_{1}, w_{2}, w_{3}, s, y_{0}, y_{1}, y_{2}, y_{3}$. We denote 
$\mathbf{w}=\left[w_{0}, w_{1}, w_{2}, w_{3}\right]^{\top}$. This subset is defined by the equations and inequalities

$$
\begin{array}{ll}
\|\mathbf{w}\|^{2}=w_{0}^{2}+w_{1}^{2}+w_{2}^{2}+w_{3}^{2}=1 & \|\mathbf{y}\|^{2}=y_{0}^{2}+y_{1}^{2}+y_{2}^{2}+y_{3}^{2}=1 \\
\mathbf{w} \cdot \mathbf{y}=w_{0} y_{0}+w_{1} y_{1}+w_{2} y_{2}+w_{3} y_{3}=0 & s \geq 0
\end{array}
$$

The modification $\widetilde{S}$ is related to $S$ via the mapping $\pi: \widetilde{S} \rightarrow S$ defined by

$$
(\mathbf{w}, s, \mathbf{y}) \longmapsto\left[w_{0} s: w_{1} s: w_{2} s: w_{3} s: y_{0}: y_{1}: y_{1}: y_{2}: y_{3}\right]
$$

The image of $\pi$ is the complement in $S$ of the 3-plane $\mathbf{y}=\mathbf{0}$ corresponding to rigid motions whose translation vector is zero. Every rigid motion with a non zero translation vector is the image by $\pi$ of two points $(\mathbf{w}, s, \mathbf{y})$ and $(-\mathbf{w}, s,-\mathbf{y})$ of $\widetilde{S}$ with $s>0$; it is easily checked that $s$ is the inverse of the length of the translation vector. So $\pi$ restricted to the set of $(\mathbf{w}, s, \mathbf{y}) \in \widetilde{S}$ with $s>0$ is just a double covering of the set of rigid motions with a non zero translation vector. The image by $\pi$ of an element $(\mathbf{w}, 0, \mathbf{y}) \in \widetilde{S}$ is the element $\left[0: 0: 0: 0: y_{0}: y_{1}: y_{1}: y_{2}: y_{3}\right]$ of the exceptional 3-plane $E$. The element $(\mathbf{w}, 0, \mathbf{y}) \in \widetilde{S}$ may be seen as the degeneration of the rigid motion associated to $(\mathbf{w}, s, \mathbf{y}) \in \widetilde{S}$ as $s$ tends to 0 , i.e. as the length of the translation vector tends to infinity. It retains the information on the rotation part in $\mathbf{w}$ and on the direction of translation in $\mathbf{t}(\mathbf{w}, \mathbf{y})$.

\section{Tsai 3-UPU}

We recall the kinematic analysis of a mobile platform with three degrees of freedom known as the Tsai 3-UPU. We rely on [6], with slight changes in presentation.

\subsection{Algebraic modelization}

The Tsai 3-UPU is a mobile platform with architecture illustrated in Figure 1. The centers $A_{i}$ and $B_{i}$ of the universal joints on the base (resp. mobile platform) form an equilateral triangle. The rotation axes 1 and 5 in the kinematic chain for each leg are tangent to the circumscribed circles. The rotation axes 2 and 4 are parallel, and both orthogonal to $\left(A_{i} B_{i}\right)$.

For the algebraic modelization, we work in the fixed frame attached to the base where the points $A_{i}$ have coordinates

$$
\mathbf{a}_{1}:\left[0, h_{1}, 0\right]^{\top} \quad \mathbf{a}_{2}:\left[0,-h_{1} / 2, \sqrt{3} h_{1} / 2\right]^{\top} \quad \mathbf{a}_{3}:\left[0,-h_{1} / 2,-\sqrt{3} h_{1} / 2\right]^{\top},
$$

where $h_{1}$ is the radius of the circle circumscribed to the base (in the computations, we shall fix $\left.h_{1}=1\right)$. The coordinates of unit vectors giving the direction of the rotation axes 1 for each leg are: 


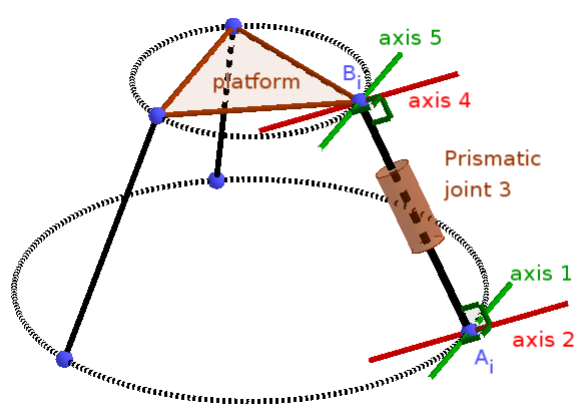

Fig. 1 A Tsai 3-UPU platform.

$$
\mathbf{v}_{1}:[0,0,1]^{\top} \quad \mathbf{v}_{2}:[0,-\sqrt{3} / 2,-1 / 2]^{\top} \quad \mathbf{v}_{3}:[0, \sqrt{3} / 2,-1 / 2]^{\top}
$$

We have a mobile frame attached to the platform where the points $B_{i}$ have coordinates $\mathbf{b}_{i}$ similar to $\mathbf{a}_{i}$ with $h_{1}$ replaced by the radius $h_{2}$ of the circle circumscribed to the platform (we shall take for short $h_{2}=h$ in the computations). The home pose of the mobile platform is when the mobile frame coincides with the fixed one. The coordinates of $B_{i}$ in the fixed frame are $\mathbf{R}(\mathbf{x}) \mathbf{b}_{i}+\mathbf{t}(\mathbf{x}, \mathbf{y})$.

The constraint equation for each leg express that the axis 1 , the line $\left(A_{i}, B_{i}\right)$ and the axis 5 are coplanar. These equations are

$$
\operatorname{det}\left(\mathbf{v}_{i}, \mathbf{R}(\mathbf{x}) \mathbf{b}_{i}+\mathbf{t}(\mathbf{x}, \mathbf{y})-\mathbf{a}_{i}, \mathbf{R}(\mathbf{x}) \mathbf{v}_{i}\right)=0 \quad \text { for } i=1,2,3
$$

After chasing denominators, these constraint equations are homogeneous equations $\left(C_{i}\right)$ of degree 4 in $\mathbf{x}, \mathbf{y}$, the Study parameters. Together with the equation $(\Sigma)$ of the Study quadric and the inequation $N_{\mathbf{x}} \neq 0$, the equations $\left(C_{i}\right)$ describe the possible poses for the mobile platform.

\subsection{The operation modes}

We form the ideal $\left\langle C_{1}, C_{2}, C_{3}, \Sigma\right\rangle$ generated by these four equations and saturate it with respect to $N_{\mathbf{x}}$ in order to get rid of parasitic components contained in the exceptional 3-plane (cf. [1] Chap. 4, § 4). We obtain a homogeneous ideal I whose projective variety is an algebraic subset of dimension 3 of the Study quadric. The operation modes of the mobile platform are the irreducible components of this algebraic set. These irreducible components are obtaine by computing the primary decomposition of $\mathfrak{I}$ (cf. [1] Chap. 4, $\S$ 8). We obtain:

- $\mathfrak{J}_{1}=\left\langle y_{0}, x_{1}, x_{2}, x_{3}\right\rangle$ : all poses obtained from the home pose by translation.

- $\mathfrak{J}_{2}=\left\langle x_{0}, y_{1}, x_{2}, x_{3}\right\rangle$ : all poses obtained by a half-turn with vertical axis followed by a translation. 
- $\mathfrak{J}_{3}=\left\langle y_{0}, y_{1}, x_{2}, x_{3}\right\rangle$ : all poses obtained by rigid motion in the base plane.

- $\mathfrak{J}_{4}=\left\langle x_{0}, x_{1}, y_{2}, y_{3}\right\rangle$ : all poses obtained by a horizontal flip followed by motion in the base plane.

- A component whose kinematic analysis is difficult. We give only the first two and the last generators of the ideal produced by the computation):

$$
\begin{aligned}
\mathfrak{J}_{5}= & \left\langle 2 x_{1} y_{1}+x_{2} y_{2}+x_{3} y_{3}, 2 x_{0} y_{0}+x_{2} y_{2}+x_{3} y_{3}, \ldots,\right. \\
& \left.x_{3} y_{0}\left(3 x_{2}^{2}-x_{3}^{2}\right)(h+1)+x_{2} y_{1}\left(3 x_{3}^{2}-x_{2}^{2}\right)(h-1)-\left(x_{2}^{2}+x_{3}^{2}\right) y_{0} y_{1}\right\rangle
\end{aligned}
$$

We shall follow the terminology of [6] and call "general mode" the fifth mode of operation.

It is remarkable that a small change in the architecture of the 3-UPU platform can drastically affect its kinematic behaviour. The SNU 3-UPU differs for the Tsai one in the disposition of axes 1 and 5: they point towards the center of the circle circumscribed to the base (resp. platform) instead of being tangent to this circle. The analysis of the operation modes of the SNU 3-UPU is done in [7]. There is an operation mode where the poses are obtained from the home pose by rotation around the origin, the operation modes $\mathfrak{J}_{1}$ to $\mathfrak{J}_{4}$ are again present, but there is no general mode $\mathfrak{J}_{5}$. Instead, there are three new components whose kinematic analysis is easy:

- $\mathfrak{J}_{6}=\left\langle x_{0}, y_{1}, y_{2}, y_{3}\right\rangle$ : all poses obtained by a half-turn with axis through origin, followed by a translation in the direction of the axis of half-turn.

- $\mathfrak{J}_{7}=\left\langle y_{0}, x_{1}, y_{2}, y_{3}\right\rangle$ : all poses obtained by the half-turn with vertical axis through origin, followed by a rigid motion of mode $\mathfrak{J}_{6}$; the rotation part is a rotation with horizontal axis.

plus a non-real component $\mathfrak{J}_{8}$ whose real points correspond to poses obtained by a translation along the vertical axis through origin, possibly composed with the halfturn around this axis. These singular poses belong also to other modes of operation.

\section{Degeneration of the general mode}

The kinematic analysis of the general operation mode of the Tsai 3-UPU is difficult. We are going to compute the boundary of this operation mode in the modification of the Study model and obtain some relevant information from this degeneration.

\subsection{The boundary of the general mode}

The projective variety $V_{5}=V\left(\mathfrak{J}_{5}\right) \subset S$ contains the poses of the general mode, plus some points of the exceptional 3-plane $E$. 
We compute the inverse image $\pi^{-1}\left(V_{5}\right)$ of $V_{5}$ in the modification $\widetilde{S}$. This is done by substituting the variables $x_{i}$ with $w_{i} s$ in the generators of $\mathfrak{J}_{5}$ and adding equations (5) to the list of generators. We obtain in this way an ideal in the ring of polynomial in variables $\mathbf{w}, s, \mathbf{y}$.

We then remove from $\pi^{-1}\left(V_{5}\right)$ the parasitic components entirely contained in the hyperplane $s=0$. This is done by saturating the ideal obtained in the preceding step with respect to $s$. This new ideal $\widetilde{\mathfrak{J}}_{5}$ describes a subset $\widetilde{V}_{5} \subset \widetilde{S}$. The points $(\mathbf{w}, s, \mathbf{y}) \in$ $\widetilde{V}_{5}$ with $s>0$ correspond to poses in the general mode $((\mathbf{w}, s, \mathbf{y})$ and $(-\mathbf{w}, s,-\mathbf{y})$ give the same pose). The points $(\mathbf{w}, 0, \mathbf{y}) \in \widetilde{V}_{5}$ may be seen as degenerate poses of the general mode, as the length of legs tends to infinity, retaining information on the rotation and the direction of translation. The intersection of $\widetilde{V}_{5}$ with the hyperplane $s=0$ form the boundary of the general mode. This boundary is a 2-dimensional algebraic subset of the boundary of the group of rigid motions.

This boundary is computed by setting $s=0$ in the ideal $\widetilde{\mathfrak{J}}_{5}$, thus obtaining an ideal in the ring of polynomials with variables $\mathbf{w}, \mathbf{y}$. The primary decomposition of this ideal reveals that the boundary of the general mode has several components:

- $\mathfrak{K}_{6}=\left\langle w_{0}, y_{1}, y_{2}, y_{3}, y_{0}-1, w_{1}^{2}+w_{2}^{2}+w_{3}^{2}-1\right\rangle$ : all degenerate poses obtained by a half-turn with axis through origin, followed by an infinite translation in the direction of the axis of half-turn.

- $\mathfrak{K}_{6^{\prime}}$, same as $\mathfrak{K}_{6}$ but with $y_{0}=-1$.

- $\mathfrak{K}_{7}=\left\langle y_{0}, w_{1}, y_{2}, y_{3}, y_{1}-1, w_{0}^{2}+w_{2}^{2}+w_{3}^{2}-1\right\rangle$ : all degenerate poses obtained by the half-turn with vertical axis through origin, followed by a a half-turn with axis through origin and then an infinite translation in the direction of the axis of the latter half-turn; the rotation part is a rotation with horizontal axis.

- $\mathfrak{K}_{7}$, same as $\mathfrak{K}_{7}$ but with $y_{1}=-1$.

There are also two non-real components $\mathfrak{K}_{8}$ and $\mathfrak{K}_{8^{\prime}}$ whose only real points are $\left(\mathbf{w}=[ \pm 1,0,0,0]^{\top}, \mathbf{y}=[0, \pm 1,0,0]^{\top}\right)$ and $\left(\mathbf{w}=[0, \pm 1,0,0]^{\top}, \mathbf{y}=[ \pm 1,0,0,0]^{\top}\right)$, corresponding to degenerate poses obtained by infinite translation in the vertical direction, possibly composed with a half-turn with vertical axis.

We have found that the boundary of the general mode of the Tsai 3-UPU decomposes as the union of the boundaries of the modes $\mathfrak{J}_{6}, \mathfrak{J}_{7}$ and $\mathfrak{J}_{8}$ of the SNU 3-UPU and that the degenerate poses in this boundary have a simple kinematic description.

\subsection{The degenerate direct kinematic problem for the general mode}

The actuated joints in the 3-UPU platform are the three prismatic joints which contol the lengths $r_{i}=A_{i} B_{i}$. The inverse kinematic mapping (IKM) for the 3-UPU platform associates to each pose the system of lengths $r_{1}, r_{2}, r_{3}$. We degenerate the IKM to the boundary of the set of poses in the modification of the Study model. We do this for the general mode of the Tsai 3-UPU. Of course, the lengths of the legs all tend to infinity, but the relevant information for the degeneration consists in the differences of the lengths of the legs. 
We obtain $r_{i}$ for $(\mathbf{w}, s, \mathbf{y}) \in \widetilde{S}$, using $\mathbf{x}=s \mathbf{w}$, as the length of the vector

$$
\frac{1}{S}\left(s \mathbf{R}(\mathbf{w}) \mathbf{b}_{i}+\mathbf{t}(\mathbf{w}, \mathbf{y})-s \mathbf{a}_{i}\right) .
$$

Recall that here $\mathbf{w}, \mathbf{y}$ and $\mathbf{t}(\mathbf{w}, \mathbf{y})$ are unit vectors. Hence

$$
r_{i}=\frac{1}{s} \sqrt{1+2 s \mathbf{t}(\mathbf{w}, \mathbf{y}) \cdot\left(\mathbf{R}(\mathbf{w}) \mathbf{b}_{i}-\mathbf{a}_{i}\right)+s^{2}\left\|\mathbf{R}(\mathbf{w}) \mathbf{b}_{i}-\mathbf{a}_{i}\right\|^{2}} .
$$

It follows that the limit of $r_{i}-r_{3}$ (for $i=1,2$ ) as $s$ tends to 0 is

$$
d_{i}=\mathbf{t}(\mathbf{w}, \mathbf{y}) \cdot\left(\mathbf{R}(\mathbf{w})\left(\mathbf{b}_{i}-\mathbf{b}_{3}\right)-\left(\mathbf{a}_{i}-\mathbf{a}_{3}\right)\right) .
$$

This formula gives $d_{1}$ and $d_{2}$ as polynomials in $\mathbf{w}$ and $\mathbf{y}$. The remainders of these polynomials by a Gröbner basis (cf. [1] Chap. $2, \S 6$ ) for the ideals $\mathfrak{K}_{6}$ and $\mathfrak{K}_{7}$ give the formulation of the degenerate IKM for these two components of the boundary of the general mode:

- for $\mathfrak{K}_{6}$, with $w_{0}=y_{1}=y_{2}=y_{3}=0, y_{0}=1$ and $w_{1}^{2}+w_{2}^{2}+w_{3}^{2}=1$ :

$$
\begin{aligned}
& d_{1}=(3 / 2)(h-1) w_{2}+(\sqrt{3} / 2)(h-1) w_{3} \\
& d_{2}=\sqrt{3}(h-1) w_{3}
\end{aligned}
$$

- for $\mathfrak{K}_{7}$, with $y_{0}=w_{1}=y_{2}=y_{3}=0, y_{1}=1$ and $w_{0}^{2}+w_{2}^{2}+w_{3}^{2}=1$ :

$$
\begin{aligned}
& d_{1}=-(\sqrt{3} / 2)(h+1) w_{2}+(3 / 2)(h+1) w_{3} \\
& d_{2}=-\sqrt{3}(h+1) w_{2}
\end{aligned}
$$

For the two real singular points of $\mathfrak{K}_{8}$, we have $d_{1}=d_{2}=0$.

These formulas are very simple: the $d_{i}$ are linear functions of $\mathbf{w}$. Hence, the degenerate direct kinematic problem (DKP) for the general mode is very easily solved. For any $\left(d_{1}, d_{2}\right)$ inside the ellipse

$$
d_{1}^{2}-d_{1} d_{2}+d_{2}^{2}=\frac{9}{4}(h-1)^{2} \quad\left(\operatorname{resp} \cdot \frac{9}{4}(h+1)^{2}\right)
$$

there are two solutions in the mode $\mathfrak{K}_{6}\left(\right.$ resp. $\left.\mathfrak{K}_{7}\right)$. For $d_{1}=d_{2}=0$ there are solutions which are singular degenerate poses.

\subsection{Comparison with the actual DKP}

The triviality of the degenerate DKP is in sharp contrast with the complexity of the actual DKP for the general mode. It is explained in [6] that the DKP for this mode is of degree 64, and that there are 24 real solutions for some values of the $r_{i}$. Due 
to this complexity, the comparison of the actual DKP with its degenerate version can only be performed partially. Nevertheless, this partial comparison shows that the degenerate DKP provides valuable information on the DKP for large lengths of legs.

We take $h=3 / 2$ and compare what happens for $d_{2}=0$ and $d_{1}$ variable and what happens for $r_{2}=r_{3}=100$ and $r_{1}$ variable. For the degenerate DKP, the easy computation gives 0 solution for $\left|d_{1}\right|>15 / 4,2$ solutions for $3 / 4<\left|d_{1}\right|<15 / 4$ and 4 solutions for $0<\left|d_{1}\right|<3 / 4$. For the DKP a heavy computation performed with the library SIROPA shows that there are 0 solution for $r_{1}-100<-3.75$.. or $r_{1}-100>$ 3.74.., 2 solutions for $-3.75 . .<r_{1}-100<-0.77$.. or $0.72 . .<r_{1}-100<3.74$.., 4 solutions for $-0.77 . .<r_{1}-100<-0.18$.. or $0.48 . .<r_{1}-100<0.72 .$. ; for $r_{1}$ closer to 100 , there are up to 12 solutions.

The separation inside the general mode between the two boundary modes $\mathfrak{K}_{6}$ and $\mathfrak{K}_{7}$ can be well seen for $r_{1}=100.6, r_{2}=r_{3}=100$. The rotation part of the four solutions to the DKP are given by $x_{0}= \pm 0.98 . ., x_{1}=0 ., x_{2}=0.15 . ., x_{3}=0$. and $x_{0}=0 ., x_{1}= \pm 0.73 . ., x_{2}=0 ., x_{3}=0.68 \ldots$

\section{Conclusion}

We have shown with the example of the Tsai 3-UPU platform that the degeneration of an operation mode whose kinematic analysis id rather complex can give a boundary whose kinematic behaviour is very easy to understand. Moreover, the degenerate DKP for this boundary gives valuable information on the original DKP, for large enough lengths of legs. This approach can also be used for other examples of parallel manipulators with restricted degrees of freedom (3-RPS, SNU 3-UPU, 4-UPU ...).

\section{References}

1. Cox, D.A., Little, J., O'Shea, D.: Ideals, Varieties and Algorithms. Springer, Cham (2015)

2. Djintelbe, N., Coste, M.: Compactification of the group of rigid motions and applications to robotics. arXiv: 1910.00319

3. Husty, M.L., Schröcker, H.P.: Algebraic geometry and kinematics. In: I.Z. Emiris, F. Sottile, T. Theobald (eds.) Nonlinear Computational Geometry, pp. 85-107. Springer New York, New York, NY (2010)

4. Selig, J.M.: Geometric Fundamentals of Robotics. Springer. Monographs in Computer Science, New York (2005)

5. Study, E.: Geometrie der Dinamen. B. G. Teubner, Leipzig (1903)

6. Walter, D., Husty, M.: Kinematic analysis of the TSAI 3-UPU parallel manipulator using algebraic methods. In: The 13th IFToMM World Congress in Mechanism and Machine Science, pp. 1-10 (2011)

7. Walter, D.R., Husty, M.L., Pfurner, M.: A complete kinematic analysis of the SNU 3-UPU parallel robot. In: D.J. Bates, G. Besana, S. Di Rocco, C.W. Wampler (eds.) Interactions of Classical and Numerical Algebraic Geometry, pp. 331-346. AMS, Providence (2009) 